\title{
Prevalence of Physical Inactivity and Sedentary Behaviors and Associations with Obesity among Saudi Adults
}

Nora A Althumiri ( $\square$ nora@althumiri.net)

Sharik Association For Health Research https://orcid.org/0000-0002-8215-6097

Nasser F BinDhim

Alfaisal University

Saleh A. Alqahtani4

Johns Hopkins University

Research article

Keywords: physical inactivity; sedentary behavior; obesity; prevalence; Saudi Arabia

Posted Date: July 14th, 2020

DOI: https://doi.org/10.21203/rs.3.rs-40306/v1

License: (c) (i) This work is licensed under a Creative Commons Attribution 4.0 International License.

Read Full License 


\section{Abstract}

Background: Worldwide, physical inactivity is ranked as the fourth leading risk factor for mortality. This study aimed to provide an update on the prevalence of physical inactivity and sedentary behavior among adults in Saudi Arabia and to examine associations with obesity.

Methods: This study was conducted as secondary analysis of a national survey that was conducted in Saudi Arabia in 2018. In this study, a proportional quota-sampling technique was used to obtain equal distributions of participants, stratified by age and gender, across the 13 regions of Saudi Arabia. Participants $(n=3,699)$ were recruited mainly via random phone interviews. The World Health Organization (WHO) recommendations (150 minutes of moderate activity/week or 75 minutes of intensive activity/week) were used to classify physical activity. Logistic regression was used for multivariate analysis.

Results: The prevalence of physical inactivity (not meeting the WHO recommendations) ranged between $66.8 \%$ and $81.2 \%$. Females and males did not differ in their frequency of physical activity. Participants spent an average of 275 minutes/day watching TV or using phone apps and 267 minutes/day doing office work. Physical inactivity was associated with increased likelihood of obesity (BMI $\geq 30$ ), as compared to those who met the WHO intensive physical activity criteria (odds ratio [OR]: $1.62 ; 95 \% \mathrm{Cl}$ : 1.23-2.13) and to those who met WHO mixed intensive/moderate physical activity criteria (OR: $1.49 ; 95 \%$ Cl: 1.24-1.81), adjusted for age and gender. However, sitting long hours for work or leisure was not significantly associated with obesity.

Conclusions: Future research is needed to identify public health interventions that would be most feasible and suitable for physical inactivity and sedentary behavior reduction at the national level for national implementation; and health policies are needed to facilitate sustainability.

\section{Introduction}

Many deaths are attributable to various chronic diseases including but not limited to hypertension, type 2 diabetes, cardiovascular disease, stroke, and obesity.(1) Findings from a recent national survey in Saudi Arabia indicated that $15 \%$ of Saudi residents have at least one chronic disease;(2) and a lack of physical activity is an underlying risk factor for several chronic diseases.(1) The minimum levels of physical activity for adults recommended by the World Health Organization (WHO) are 150 minutes of moderate body movement per week or intensive body movement for at least 75 minutes per week; and sedentary behavior is defined as waking activity characterized by long periods of sitting or lying down. $(3,4)$ Physical inactivity has been described as a global health problem, it is associated with the development of non-communicable diseases (NCDs), and it is the fourth leading risk factor for mortality globallyaccounting for an estimated $6 \%$ of deaths.(4) The Centers for Disease Control and Prevention (CDC) reported that only half of adults and a third of youth in the United States meet physical activity guidelines and recommendations.(5) 
People who are physically active live longer and have lower risks for depression, stroke, type 2 diabetes, and obesity.(6) Recommendations such as healthy lifestyle changes that include healthy dieting and increased physical activity are known to improve overall health, especially for those with prior and current medical conditions.(6) Physical activity guidelines for Americans recommend that adults should achieve at least 2 hours and 30 minutes ( 150 Minutes) a week of moderate-intensity aerobic physical activity.(5, 6) However, for more extensive health benefits, guidelines recommend adults to increase their physical activity to 5 hours a week (300 minutes) of moderate intensity.(5-7)

According to the World Health Organization (WHO), the Eastern Mediterranean Region has the highest prevalence of physical inactivity (35\%);(8) and Saudi Arabia has the highest rate of physical inactivity among the Gulf Cooperation Countries.(9) In particular, WHO reported that $58.8 \%$ of Saudi adults were considered physically inactive,(10) and WHO's estimate of physical inactivity among Saudi adults was similar to an estimate from a national survey $(60 \%)$ that was conducted by the Ministry of Health in 2013. (9) In terms of gender, several national household cross-sectional estimates of physical inactivity range between $44.5 \%-66.6 \%$ and $72.9 \%-78.1 \%$ for males and females, respectively.(11) However, few studies have characterized sedentary behavior in Saudi Arabia mainly for segmented samples not on national level. $(12,13)$ In addition, more studies are needed to characterize physical inactivity in Saudi Arabia and associations with obesity.(14) The objectives of this study were to estimate the prevalence of physical inactivity and to characterize sedentary behavior duration and frequency among adults in Saudi Arabia, and to examine associations between physical activity and obesity and between sedentary behavior and obesity.

\section{Methods}

\section{Study Design and Sampling Strategy}

This study conducted a secondary analysis of raw data from a nationwide cross-sectional study that was conducted between March and August 2018 in Saudi Arabia. The full descriptive results and methodology of the original study can be found elsewhere in a published governmental report.(2) However, an in-depth summary of the original study's methodology will be described. A proportional quota sampling technique was employed to acquire an equal distribution of participants stratified by age and gender across the 13 regions of Saudi Arabia.

Two age groups based on the Saudi Arabia median age of 37 years were used leading to a quota of 52 . The QPlatform ${ }^{\circledR}$ data collection system, which had integrated eligibility and sampling modules, was used to control the distribution of the sample.(15) The eligibility module included three questions that were used to determine the completeness of the sampling quota, including age, gender, and region. To compare age and gender across regions, the sample size was calculated based on a medium effect size of approximately 0.3 with $80 \%$ power and $95 \%$ confidence level.(16) Thus, each quota required 70 participants, and the targeted total sample was 3,640 participants.

\section{Participant Recruitment}


Participant recruitment was limited to Arabic-speaking Saudi residents $\geq 18$ years old. To identify potential participants, a random phone number list was generated from the Sharik Association for Health Research.(17) The Sharik database is composed of individuals who are interested in participating in health research. Participants were contacted by phone on up to three occasions. Where quotas could not be filled by telephone (e.g., remote community, cultural factors), participants were recruited by convenience face-to-face interviews in public places, such as shopping malls. A total of 3,699 participants provided informed consent and participated in the study-approximately $25 \%$ and $75 \%$ of participants completed face-to-face and phone interviews, respectively.

\section{Questionnaire}

To assess physical activity, a survey questionnaire was adopted from questions on the 2016-2017 National Health Interview Survey (NHANES).(18) Questions included sociodemographic characteristics, height and weight, number of minutes of exercise and the frequency of preforming high and moderate intensity exercise, the number of hours spent watching TV, playing video games or using phone apps, and the number of sitting hours doing office work.

Linguistic validation was done to ensure that the translation from English to Arabic had the same intention as questions from the original source questionnaire. The standard backward and forward translation was done. Two nutritionists and one research professional independently conducted the forward translation, and the backward translation was done separately by two professional translators. A focus group of eight participants was asked to discuss and answer the questionnaire. To test the questionnaire's reliability, this process was repeated a second time with the same people. Afterward, 40 participants were interviewed by phone to ensure the accuracy of the original meaning and to better understand the questions. According to the results of the pilot study and feedback from the researchers and interviewers, the questionnaire was edited further and the final version of the questionnaire was produced. All questions had to be answered for the responses to be successfully submitted to the database. All data were coded and stored on the QPlatform database.(15) The Saudi Food and Drug Authority Ethics Committee (SFDA18-0004) approved the research protocol.

\section{Primary Outcomes of Interest}

The primary outcomes of interest for this study were prevalence of physical inactivity and characterization of sedentary behavior duration and frequency, and associations between physical inactivity and obesity and between sedentary behavior and obesity.

\section{Outcome Measures}

To categorize participants, this study used WHO's recommended physical activity guidelines for adults (18-64 years old) - which are in line with guidelines from the CDC and the American Heart Association $(\mathrm{AHA})(4,5): 1)$ intensive physical activity, 75 minutes of intensive physical activity per week, 2) moderate physical activity, 150 minutes of moderate physical activity per week; and 3) combination of intensive and moderate physical activity, 150 minutes of an equivalent combination of intensive and moderate 
physical activity per week. From self-reported responses on the questionnaire (i.e. number of exercise minutes and frequency of intensity level per week), participants who met WHO recommended guidelines were defined as meeting physical activity guidelines and participants who did not met WHO recommended guidelines were defined as not meeting physical activity guidelines (physical inactivity). Participants' height and weight were used to calculate their body mass index (BMI). Participants with $\mathrm{BMI} \geq 30$ were defined as obese.(19) The number of leisure sitting hours and office sitting hours that participants reported were used to quantify sedentary behavior.

\section{Data Analysis}

Measures of central tendency and frequency distributions were used to characterize the sample. Chisquare was used to compare males and females on dichotomous variables of physical activity. Logistic regression was used to assess associations between physical activity and obesity and between sedentary behavior and obesity, adjustment for age and gender were preformed. All statistical analyses were performed using IBM SPSS Version 25.0.

\section{Results}

\section{Demographic Characteristics}

The mean age of participants was 38 (11.5) years (mean, standard deviation) (Table 1). Proportions of males and females were nearly equal ( $51.2 \%$ vs. $48.8 \%$, respectively). The majority of participants had a college education $\geq$ a bachelor's degree $(52.7 \%)$, and the majority were currently working $(74.4 \%)$ and married $(65.0 \%)$. Nearly a third were obese $(31.6 \%)$. 
Table 1

Participant Characteristics

\begin{tabular}{|c|c|}
\hline Characteristics & $\mathrm{N}(\%) / \mathrm{M}(\mathrm{SD})$ \\
\hline \multicolumn{2}{|l|}{$N=3,699$} \\
\hline Gender & $1,893(51.2)$ \\
\hline Male & $1,806(48.8)$ \\
\hline \multicolumn{2}{|l|}{ Female } \\
\hline Age & $38(11.5)$ \\
\hline Education & $1,750(47.3)$ \\
\hline < Bachelor's degree & $1,949(52.7)$ \\
\hline \multicolumn{2}{|l|}{$\geq$ Bachelor's degree } \\
\hline Work Status & $2,752(74.4)$ \\
\hline Currently working & $917(24.9)$ \\
\hline \multicolumn{2}{|l|}{ Currently not working } \\
\hline Current Martial Status & $1,294(35.0)$ \\
\hline Single & $2,405(65.0)$ \\
\hline \multicolumn{2}{|l|}{ Married } \\
\hline Monthly Income & $639(17.3)$ \\
\hline$<5,000 \mathrm{SR}$ & $854(23.1)$ \\
\hline Between $5,001-10,000$ SR & $776(21.0)$ \\
\hline Between $10,001-15,000$ SR & $806(21.8)$ \\
\hline$\geq 15,000 \mathrm{SR}$ & $624(16.8)$ \\
\hline \multicolumn{2}{|l|}{ Refused to answer } \\
\hline BMI Category & $165(4.5)$ \\
\hline Underweight & 1,159 (31.3) \\
\hline Normal & $1,205(32.6)$ \\
\hline Overweight & $1,170(31.6)$ \\
\hline \multicolumn{2}{|l|}{ Obese } \\
\hline SR, Saudi Riyal & \\
\hline
\end{tabular}

Physical Activity and Bivariate Comparisons 
Only 33.5\% of participants met WHO's recommendations for moderate physical activity (150 min/week), $18.8 \%$ met WHO's recommendations for intensive physical activity ( $75 \mathrm{~min} /$ week), and $28.1 \%$ met the recommended weekly combined amount of intensive and moderate physical activity (150 min/week) (Table 2). The average time spent engaging in physical activity was 53 (1.15) minutes per activity day. In bivariate analysis, females and males did not differ in in meeting WHO recommendations; moderate, intensive, or combined intensive and moderate physical activity.

Table 2

Bivariate Comparisons of Gender on Physical Activity

\begin{tabular}{|c|c|c|c|c|}
\hline Characteristics & $\begin{array}{l}\text { Sample N } \\
(\%)\end{array}$ & $\begin{array}{l}\text { Female, N } \\
\text { (\%) }\end{array}$ & $\begin{array}{l}\text { Male, N } \\
(\%)\end{array}$ & $\begin{array}{l}\mathrm{P} \text { - } \\
\text { value }\end{array}$ \\
\hline Moderate Physical Activity & & & & 0.48 \\
\hline Not as Recommended & $\begin{array}{l}2,460 \\
(66.5)\end{array}$ & $\begin{array}{l}1,191 \\
(48.4)\end{array}$ & $\begin{array}{l}1,269 \\
(51.6)\end{array}$ & \\
\hline As Recommended & $\begin{array}{l}1,239 \\
(33.5)\end{array}$ & $623(50.3)$ & $\begin{array}{l}616 \\
(49.7)\end{array}$ & \\
\hline Intensive Physical Activity & & & & 1.00 \\
\hline Not as Recommended & $\begin{array}{l}3,002 \\
(81.2)\end{array}$ & $\begin{array}{l}1,467 \\
(48.9)\end{array}$ & $\begin{array}{l}1,535 \\
(51.1)\end{array}$ & \\
\hline As Recommended & $697(18.8)$ & $340(48.8)$ & $\begin{array}{l}357 \\
(51.2)\end{array}$ & \\
\hline $\begin{array}{l}\text { Combination of Intensive and Moderate Physical } \\
\text { Activity }\end{array}$ & & & & 0.86 \\
\hline Not as Recommended & $\begin{array}{l}2,661 \\
(71.9)\end{array}$ & $\begin{array}{l}1,297 \\
(48.7)\end{array}$ & $\begin{array}{l}1,364 \\
(51.3)\end{array}$ & \\
\hline As Recommended & $\begin{array}{l}1,038 \\
(28.1)\end{array}$ & $528(50.9)$ & $\begin{array}{l}510 \\
(49.1)\end{array}$ & \\
\hline
\end{tabular}

\section{Sedentary Behavior}

The average number of hours of leisure sitting (e.g. watching TV, playing video games, or using phone apps) was 4.58 (3.5) hours/day or 275 minutes/day (Table 3). The average number of hours of sitting doing office work was 4.40 (3.1) hours/day or 264 minutes/day. Of those who met criteria of being physically active, between $45.7 \%$ and $46.9 \%$ spent more than 4 hours/day of leisure sitting. 
Table 3

Sedentary Behavior Duration and Frequency: Leisure and Office Sitting Time

\begin{tabular}{|ll|}
\hline Sedentary Behavior Duration and Frequency & N (\%)/Mean (SD) \\
\hline Number of Per Day Leisure Sitting Hours & $4.58(3.5)$ \\
\hline Less than 2 hours/day & $1065(28.8)$ \\
\hline 2 hours to 4 hours/day & $866(23.4)$ \\
\hline 4 hours to 6 hours/day & $628(17.0)$ \\
\hline More than 6 hours/day & $1140(30.8)$ \\
\hline Number of Per Day Sitting Hours Doing Office Work & $4.40(3.1)$ \\
\hline Less than 2 hours/day & $946(25.6)$ \\
\hline 2 hours to 4 hours/day & $416(11.2)$ \\
\hline 4 hours to 6 hours/day & $343(9.3)$ \\
\hline More than 6 hours/day & $1994(53.9)$ \\
\hline Leisure sitting hours included watching TV, playing video games, or using phone apps \\
\hline OCiations between Physical Activity and Obesity and \\
Veen Sedentary Behavior and Obesity
\end{tabular}

In multivariate analysis, adjusting for gender and age, not meeting WHO's recommendations of moderate (odds ratio [OR]: 1.44; 95\% Cl: 1.18-1.76), intensive (OR:1.62; 95\% Cl: 1.23-2.13), and combined intensive and moderate (OR: $1.49 ; 95 \% \mathrm{Cl}: 1.24-1.81)$ physical activity were positively associated with obesity (Table 4). However, there were no statistically significant associations between sedentary behavior and obesity (table not shown). 
Table 4

Logistic Regression Examining Associations between Physical Activity and Obesity

\begin{tabular}{|c|c|c|}
\hline Variables & $\begin{array}{l}\text { Crude OR (95\% Cl) (p- } \\
\text { value) }\end{array}$ & $\begin{array}{l}\text { Adjusted OR (95\% Cl) (p- } \\
\text { value)* }\end{array}$ \\
\hline Moderate Physical Activity & Reference & Reference \\
\hline As Recommended & \multirow[t]{2}{*}{$\begin{array}{l}1.42(1.18-1.72) \\
(0.001)\end{array}$} & \multirow[t]{2}{*}{$1.44(1.18-1.76)(0.001)$} \\
\hline Not as Recommended & & \\
\hline Intensive Physical Activity & Reference & Reference \\
\hline As Recommended & \multirow{2}{*}{$\begin{array}{l}1.74(1.35-2.25) \\
(0.001)\end{array}$} & \multirow[t]{2}{*}{$1.62(1.23-2.13)(0.001)$} \\
\hline Not as Recommended & & \\
\hline Combination of Intensive and Moderate & Reference & Reference \\
\hline As Recommended & \multirow[t]{2}{*}{$\begin{array}{l}1.48(1.24-1.77) \\
(0.001)\end{array}$} & \multirow[t]{2}{*}{$1.49(1.24-1.81)(0.001)$} \\
\hline Not as Recommended & & \\
\hline $\begin{array}{l}\text { Moderate Physical Activity: ( } \geq 30 \text { minute } \\
\text { minutes/day/week); OR, odds ratio; } \mathrm{Cl} \text {, c }\end{array}$ & $\begin{array}{l}\text { eek); Intensive Physical } \\
\text { e interval. }\end{array}$ & ctivity $(\geq 20$ \\
\hline
\end{tabular}

\section{Discussion}

Using national survey data from a sample of 3,699 Saudi adult participants, this study estimated the prevalence of physical inactivity and characterized sedentary behavior duration and frequency in Saudi Arabia, and examined associations between physical activity and obesity and between sedentary behaviors and obesity. Several main findings emerged from the study. First, males and females did not differ in their frequency of physical activity. This finding is in contrast with a systematic review by AlHazzaa et al. 2018 who found that Saudi females were less likely to be physically active than males.(11) The inconsistency could be due to the current study's recruitment of participants from all 13 regions of Saudi Arabia; whereas, most of the studies in the review by Al-Hazzaa and colleagues did not recruit participants across all 13 regions of Saudi Arabia. Additionally, females were permitted access to gyms in 2017, and more gyms are currently open across Saudi Arabia. In addition, in 2017 physical activity classes were introduced in female school.

Second, our findings indicated that $66.8-81.2 \%$ of Saudi adults met criteria for physical inactivity or not meeting the WHO recommendations. This finding is consistent with the high prevalence of physical inactivity that was found in previous studies conducted in university students and hospital settings.(2) $(10,11)$ The General Authority for Statistics (GAS) released an annual report of physical activity prevalence in 2018.(20) Although the GAS used different sampling methodology and question phrasing 
and a lower minimum age requirement for study inclusion ( 15 years vs. 18 years old), only $17.40 \%$ of Saudis met WHO's recommended level of physical activity, which was relatively similar to the findings from this study.

Moreover, we found that Saudi adults spent an average of 275 minutes per day engaged in sedentary behavior. Globally, this number is considered to be one of the highest.(7) Thus, more health promotion campaigns are needed in Saudi Arabia to increase public awareness of the consequences of sedentary behavior and the health benefits of physical activity. In addition, participants who did not meet WHO's recommended amount of weekly physical activity (i.e. who were physically inactive) had increased odds of obesity. One national study in 2013 found similar results among men but not women because of low overall physical activity among women in the sample.(21) However, studies outside of Saudi Arabia found that physical inactivity was independently associated with increased odds of obesity among adults-as well as among adolescents. $(22,23)$ On the other hand, sedentary behavior was not associated with obesity in this study. This might be due to a large proportion of sedentary participants were concurrently physically active-a phenomenon known as the Active Couch Potato (identified in the AusDiab TV-time studies),(24, 25)

This study had notable limitations and strengths. The study was limited to an analysis of cross-sectional data. Quota sampling instead of random sampling was used for participant recruitment which has a risk of selection bias. Notwithstanding, the use of quota sampling allowed for recruitment of a balanced study sample in terms of gender and age. Data integrity checks, inherent to the QPlatform data collection system, minimized data entry of invalid or erroneous data. Linguistic validation and questionnaire piloting were employed to strengthen the questionnaire's reliability. The study analyzed a large study sample with high representativeness of adults in Saudi Arabia.

\section{Conclusion}

The prevalence of physical inactivity in Saudi Arabia was alarmingly high. Health education efforts are needed to educate Saudi adults of the harmful health effects of physical inactivity; and health promotion campaigns are needed to promote lifestyle changes that reduces the frequency of physical inactivity and sedentary behavior. Future research is needed to identify public health interventions that would be most feasible and suitable for physical inactivity and sedentary behavior reduction at the national level for national implementation; and health policies are needed to facilitate sustainability. Otherwise, it is probable that current rates of physical inactivity among Saudi adults will incrementally lead to significant increases in chronic medical conditions and associated morbidity and mortality as they age in the coming years.

\section{Declarations}

Ethics approval and consent to participate 
The Saudi Food and Drug Authority Ethics Committee (SFDA18-0004) approved the research protocol and consent obtained verbally during the interview

Availability of data and materials

Available from the corresponding author upon request

Competing interests

None

Funding

None

Authors' contributions

NAA, NFB, SAA participated in the conceptual design and formulation of the research questions, and development and review of the manuscript. NAA \& NFB analyzed the data.

Acknowledgements

None

\section{References}

1. Petrie JR, Guzik TJ, Touyz RM. Diabetes, hypertension, and cardiovascular disease: clinical insights and vascular mechanisms. Can J Cardiol. 2018;34(5):575-84.

2. Althumiri NA, Alammari NS, Almubark RA, Alnofal FA, Alkhamis DJ, Alharbi LS, et al. The National Survey of Health, Diet, Physical Activity and Supplements among Adults in Saudi Arabia. Food Drug Regulatory Science Journal. 2018;1(1):1-.

3. van der Ploeg HP, Hillsdon M. Is sedentary behaviour just physical inactivity by another name? International Journal of Behavioral Nutrition Physical Activity. 2017;14(1):142.

4. World Health Organization. Global recommendations on physical activity for health: World Health Organization; 2010.

5. $\mathrm{CDC} /$ National Center for Health Statistics. Exercise or Physical Activity [Available from: https://www.cdc.gov/nchs/fastats/exercise.htm.

6. Willett WC, Koplan JP, Nugent R, Dusenbury C, Puska P, Gaziano TA. Prevention of chronic disease by means of diet and lifestyle changes. Disease Control Priorities in Developing Countries 2nd edition: The International Bank for Reconstruction and Development/The World Bank; 2006.

7. Caspersen CJ, Powell KE, Christenson GM. Physical activity, exercise, and physical fitness: definitions and distinctions for health-related research. Public health rep. 1985;100(2):126-31. 
8. World Health Organization. Prevalence of insufficient physical activity [Available from: https://www.who.int/gho/ncd/risk_factors/physical_activity_text/en/.

9. Al-Zalabani AH, Al-Hamdan NA, Saeed AA. The prevalence of physical activity and its socioeconomic correlates in Kingdom of Saudi Arabia: A cross-sectional population-based national survey. Journal of Taibah University Medical Sciences. 2015;10(2):208-15.

10. Al-Hazzaa HM. The public health burden of physical inactivity in Saudi Arabia. J Fam Commun Med. 2004;11(2):45.

11. Al-Hazzaa HM. Physical inactivity in Saudi Arabia revisited: a systematic review of inactivity prevalence and perceived barriers to active living. International journal of health sciences. 2018;12(6):50.

12. Alhakbany MA, Alzamil HA, Alabdullatif WA, Aldekhyyel SN, Alsuhaibani MN, Al-Hazzaa HM. Lifestyle habits in relation to overweight and obesity among Saudi women attending Health Science Colleges. Journal of epidemiology global health. 2018;8(1):13-9.

13. Al-Hazzaa HM, Abahussain NA, Al-Sobayel HI, Qahwaji DM, Musaiger AO. Physical activity, sedentary behaviors and dietary habits among Saudi adolescents relative to age, gender and region. International Journal of Behavioral Nutrition Physical Activity. 2011;8(1):140.

14. Albawardi NM, Jradi H, Almalki AA, Al-Hazzaa HM. Level of sedentary behavior and its associated factors among saudi women working in office-based jobs in Saudi Arabia. Int J Environ Res Public Health. 2017;14(6):659.

15. NF BinDhim. Smart Health Project 2012 [Available from: https://shproject.net/.

16. Cohen J. Statistical power analysis for the behavioral sciences: Academic press; 2013.

17. Sharik Association for Health Research (SharikHealth). 2015.

18. Statistics NCfH. National health and nutrition examination survey Centers for Disease Control and Prevention growth charts: United States. US Department of Health and Human Services. www cdc gov/nchs/about/major/nhanes/growthcharts/charts htm. 2008.

19. Healthy Weight. About Adult BMI [Available from:

https://www.cdc.gov/healthyweight/assessing/bmi/adult_bmi/index.html.

20. Saudi General Authority for Statistics. Bulletin of Household Sport Practice Survey 2018 [Available from: https://www.stats.gov.sa/sites/default/files/household_sport_practice_survey_2018_en.pdf.

21. Memish ZA, El Bcheraoui C, Tuffaha M, Robinson M, Daoud F, Jaber S, et al. Peer reviewed: obesity and associated factors-Kingdom of Saudi Arabia, 2013. Preventing chronic disease. 2014;11.

22. Martínez-González M, Martinez JA, Hu F, Gibney M, Kearney J. Physical inactivity, sedentary lifestyle and obesity in the European Union. Int J Obes. 1999;23(11):1192-201.

23. Pietiläinen KH, Kaprio J, Borg P, Plasqui G, Yki-Järvinen H, Kujala UM, et al. Physical inactivity and obesity: a vicious circle. Obesity. 2008;16(2):409-14.

24. Owen N, Healy GN, Matthews CE, Dunstan DW. Too much sitting: the population-health science of sedentary behavior. Exerc Sport Sci Rev. 2010;38(3):105. 
25. Healy GN, Wijndaele K, Dunstan DW, Shaw JE, Salmon J, Zimmet PZ, et al. Objectively measured sedentary time, physical activity, and metabolic risk: the Australian Diabetes, Obesity and Lifestyle Study (AusDiab). Diabetes Care. 2008;31(2):369-71. 